\title{
COVID-19 y tiroides. Revisión de la literatura y recomendaciones para pacientes con enfermedad tiroidea
}

\author{
Thyroid and COVID-19. Review and recommendations for \\ patients with thyroid disease
}

\author{
Victor M. Blanco', Natalia Aristizabal-Henao², Carolina Aguilar-Londoño, José Luis Torres- \\ Grajales 4 CvLAC
}

Fecha correspondencia: Recibido: mayo 20 de 2020. Revisado: julio 21 de 2020. Aceptado: julio 28 de 2020.

Forma de citar: Blanco VM, Aristizabal-Henao N, Aguilar-Londoño C, TorresGrajales JL. COVID-19 y tiroides. Revisión de la literatura y recomendaciones para pacientes con enfermedad tiroidea. Rev CES Med. 2020; Especial COVID-19: 78-85.

\section{Open access}

(C) Derecho de autor Licencia creative commons

Ética de publicaciones

Revisión por pares

Gestión por Open Journal System DOl: http://dx.doi.org/10.21615/ cesmedicina.34.COVID-19.11 ISSN 0120-8705 e-ISSN 2215-9177

Comparte

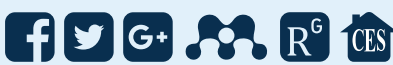

\section{Resumen}

Las enfermedades de la tiroides, tanto benignas como malignas, son altamente prevalentes a nivel mundial, por lo que es muy probable que durante la pandemia por SARS-CoV-2 veamos pacientes con ambas enfermedades. Esto exige el conocimiento de las implicaciones potenciales de este nuevo virus en el funcionamiento de la glándula, en los tratamientos usuales para estas enfermedades y en consideraciones especiales para este grupo poblacional. A la fecha no hay evidencia que soporte que las enfermedades tiroideas aumenten el riesgo de infección o severidad de la enfermedad; sin embargo, es posible que durante infecciones severas por SARS-CoV-2 en personas con o sin antecedente de enfermedad tiroidea puedan presentar alteración de las pruebas tiroideas, aunque transitoriamente y sin requerimiento de tratamiento específico. Es fundamental que los pacientes continúen con sus tratamientos ambulatorios y se difiera, en la medida de lo posible, los procedimientos quirúrgicos o la administración de yodo radioactivo hasta que se considere seguro realizarlos.

Palabras clave: COVID-19; SARS-CoV-2, Hipotiroidismo; Hipertiroidismo; Cáncer de tiroides.
Abstract
Both benign and malignant thyroid diseases are highly prevalent worldwide, so it is highly likely that during the COVID-19 pandemic we will see patients with this comorbidity. This requires knowledge of the potential implications of this new virus in the functioning of the gland, the usual treatments for these diseases and special recommendations in this population. To date, there is no evidence to support that thyroid diseases increase the risk of infection or the disease severity. However, it is possible that during se- vere SARS-CoV-2 infections in people with or without history of thyroid disease, the thyroid tests may be altered, although transitory and does not require specific treatment. It is essential for patients to continue with their outpatient treatments and defer as far as possible surgical procedures or administration of radioactive iodine until it is considered safe to perform. 


\section{Sobre los autores:}

1. Médico Internista, Fellow de Endocrinología. Universidad Pontificia Bolivariana. Medellín, Colombia.

\section{Médica Internista, Endocrinóloga. Clínica Las Américas. Docente Universidad CES. Docente Universidad Pontificia Bolivariana. Medellín, Colombia.}

3. Médica Internista, Endocrinóloga. Clínica Las Américas. Medellín, Colombia.

4. Médico Internista, Endocrinólogo. Clínica Las Américas, Endocrino Centro de Estudios Hormonales S.A., Docente Universidad Pontificia Bolivariana.

En una cohorte retrospectiva con 274 pacientes infectados por SARS-CoV-2 y sin antecedentes de enfermedad tiroidea, se compararon 161 pacientes recuperados con 113 fallecidos, observando concentraciones significativamente más bajas de TSH (tirotropina) y T3 (triyodotironina) libre en los pacientes fallecidos.
Keywords: COVID-19; SARS-CoV-2; Hypothyroidism, Hyperthyroidism; Thyroid cancer.

\section{Introducción}

La pandemia actual por coronavirus inició el 8 de diciembre de 2019 en la ciudad de Wuhan en China con la inusual presentación de varios pacientes con neumonía severa de causa no identificada hasta ese momento. Estudios de genómica identificaron un nuevo virus que fue denominado SARS-CoV-2 por tener una similitud genómica del $70-75 \%$ con el coronavirus que causó la pandemia del síndrome respiratorio agudo severo (SARS) del 2002-2003. El 11 de febrero de 2020 la Organización Mundial de la Salud le da el nombre de COVID-19 a la enfermedad causada por el SARS-CoV-2 y finalmente la declara pandemia global el 11 de marzo de 2020. En Colombia, el primer caso reportado fue el 6 de marzo de 2020 y desde entonces el número de personas infectadas viene en ascenso. El escaso conocimiento científico sobre esta emergente enfermedad COVID-19 impone retos para el manejo de los pacientes, muchos de los cuales tendrán comorbilidades tiroideas benignas o malignas dada su alta prevalencia en nuestra población. Esta situación exige el conocimiento de las implicaciones potenciales de este nuevo virus en el funcionamiento de la glándula tiroides y la necesidad de tener consideraciones especiales en este grupo poblacional.

\section{SARS-CoV-2 y tiroides}

Diferentes virus se han asociado con tiroiditis. Hay evidencia directa de infección por paperas y virus espumoso humano (HFV) con tiroiditis subaguda, HTLV-I, rubéola, herpesvirus, parvovirus y Epstein-Barr virus (EBV) con tiroiditis autoinmune de Hashimoto, y el HTLV-I, VIH y HFV con enfermedad de Graves (1).

Un meta-análisis de la prevalencia de comorbilidades y sus efectos en 1576 pacientes chinos infectados con SARS-CoV-2, reporta que la HTA (21,1\%), diabetes (9,7\%), enfermedad cardiovascular $(8,4 \%)$ y neumopatías $(1,5 \%)$ podrían ser factores de riesgo para enfermedad severa sin mención de enfermedad tiroidea (2). Se reportaron enfermedades endocrinas en $8 \%$ de los hospitalizados con COVID-19 sin especificar enfermedad tiroidea (3). En India, se reportó una prevalencia de hipotiroidismo del 4,7 \% en una serie de 21 pacientes (4).

Con respecto a las alteraciones de las pruebas de función tiroidea y SARS-CoV-2, un metaanálisis con 3377 pacientes evalúa la asociación entre parámetros de laboratorio y la severidad y mortalidad por COVID-19 y concluye que en pacientes hospitalizados con distrés respiratorio, el recuento de leucocitos, linfocitos, plaquetas, los niveles de IL-6 y ferritina sérica fueron potenciales marcadores de progresión a enfermedad severa; sin embargo, los niveles de hormonas tiroideas no fueron evaluados (5).

En una cohorte retrospectiva con 274 pacientes infectados por SARS-CoV-2 y sin antecedentes de enfermedad tiroidea, se compararon 161 pacientes recuperados con 113 fallecidos, observando concentraciones significativamente más bajas de TSH (tirotropina) y T3 (triyodotironina) libre en los pacientes fallecidos. Si bien la T4 (tiroxina) también tuvo un comportamiento similar, no fue estadísticamente significativo (6). Hallazgos similares fueron reportados durante la pandemia del SARS-CoV de 2002-2003: un estudio comparó el perfil tiroideo de 48 pacientes con SARS-CoV versus 37 controles, evidenciando concentraciones significativamente más bajas de TSH, T3 y T4, principalmente en enfermos en fase aguda (7). En 2004, se publicó una serie de cuatro autopsias de pacientes fallecidos por SARS-CoV donde se demostró, a través de pruebas de biología molecular, la infección directa del SARS-CoV en los 
Hasta el momento, no existe evidencia de la relación causal entre la infección por SARS-CoV-2 con enfermedad tiroidea y tampoco de la asociación del antecedente de enfermedad tiroidea y un mayor riesgo de infección, severidad o mortalidad por SARS-CoV-2. pulmones (50-75\% de células infectadas) y en adrenales y paratiroides (25-50\% de células infectadas) en menos del $25 \%$ de las células de la hipófisis, pero no se identificó ARN del SARS-CoV en la tiroides (8).

Se han reportado cambios en la estructura de las células foliculares y parafoliculares de la tiroides, compatibles con un extenso daño celular con destrucción del epitelio (9). Si bien no es evidencia directa de infección por el SARS-CoV en la glándula, estos hallazgos histológicos podrían soportar cambios inflamatorios en la tiroides en pacientes fallecidos a causa de la infección.

Los resultados de inmunohistoquímica de las adenohipófisis en autopsias de fallecidos por SARS-CoV reportan una disminución significativa en la expresión de TSH, $\mathrm{GH}$ y ACTH, así como un aumento de la expresión de prolactina, FSH y LH en células hipofisiarias comparativamente con controles no infectados (10). Esta alteración hipofisiaria que se presenta en algunos pacientes también fue reportada en otro estudio de 58 pacientes infectados por SARS-CoV con una disminución significativa en los niveles séricos de prolactina, LH y FSH (11).

En este sentido, un estudio prospectivo durante la pandemia de SARS siguió durante un año a 61 pacientes recuperados y sin evidencia de endocrinopatía preexistente, con mediciones de TSH, T3L, T4L, cortisol sérico (8 a.m.), ACTH, cortisol libre en orina de 24 horas y DHEAS al momento del alta hospitalaria (basal) y cada tres a seis meses, mostrando $39 \%$ de hipocortisolismo, 6,6 \% de hipotiroidismo y 3,3\% de hipertiroidismo subclínico al momento basal. Estas alteraciones fueron transitorias, con recuperación de la función entre los tres y nueve meses sin necesidad de suplencia hormonal; razón por la cual los autores sugieren un posible papel del SARS-CoV como causa de una hipofisitis reversible o efecto hipotalámico directo, alterándose con mayor frecuencia el eje hipotálamo-hipófisis-adrenal que el eje hipotálamo-hipófisis-tiroides (12). Sin embargo, estas alteraciones hormonales también podrían corresponder a un síndrome enfermo post viral ("sickness syndrome") o síndrome de eutiroideo enfermo dependiendo del eje comprometido.

En el síndrome enfermo post viral, incluido post-SARS, hay una activación de la respuesta inmune e inflamatoria que conduce a la producción o circulación periférica de agentes proinflamatorios, principalmente NF-KB que, a través de señales aferentes endocrinas o neurales, estimulan una cascada inflamatoria del sistema nervioso central responsable de los efectos en el eje hipotálamo-hipófisis-adrenal (13), a las cuales contribuye además el uso de esteroides exógenos en los casos más severos. Tanto el síndrome enfermo post viral como el síndrome de eutirodeo son estados adaptativos para conservar energía en pacientes sometidos a gran estrés, que son transitorios y en principio no requieren suplencia hormonal.

Considerando lo anterior puede decirse que, hasta el momento, no existe evidencia de la relación causal entre la infección por SARS-CoV-2 con enfermedad tiroidea y tampoco de la asociación del antecedente de enfermedad tiroidea y un mayor riesgo de infección, severidad o mortalidad por SARS-CoV-2. Las posibles alteraciones del eje hipotálamo-hipófisis-tiroides asociadas a COVID-19 siguen siendo materia de estudio, pudiendo explicarse por la ocurrencia de hipofisitis o tiroiditis con disfunción hormonal reversible o de alteraciones tiroideas como respuesta adaptativa a enfermedad grave (síndrome del eutiroideo enfermo), que son también transitorias sin requerir intervenciones adicionales. 


\section{Recomendaciones para enfermedades tiroideas en tiempos de COVID-19}

Basados en la limitada evidencia disponible, grupos de expertos como la Asociación Americana de Tiroides, la Sociedad Española de Endocrinología y Nutrición y la Fundación Británica de Tiroides, han publicado en sus respectivas páginas web una serie de recomendaciones dirigidas a pacientes con enfermedad tiroidea, las cuales se presentan y resumen a continuación. Se debe tener en cuenta que estas recomendaciones se basan principalmente en la opinión de expertos, por tanto, debe entenderse que todas las decisiones deben tomarse caso por caso considerando factores individuales del paciente y los recursos sanitarios locales y regionales disponibles.

\section{Enfermedad tiroidea autoinmune (tiroiditis de Hashimoto o enfermedad de Graves)}

Hasta el momento, no hay evidencia de que los pacientes con enfermedades tiroideas autoinmunes tengan una mayor predisposición a la infección por COVID-19 o que su enfermedad sea más grave, pero considerando que el hipotiroidismo es muy frecuente, no es de extrañar que sea una de las comorbilidades encontradas en los

Aunque no se ha demostrado que las personas con disfunción tiroidea descompensada tengan mayor riesgo de infecciones virales, es posible que en casos de disfunción tiroidea severa no controlada aumenten las complicaciones; por lo tanto, se recomienda que los pacientes con enfermedad tiroidea continúen tomando su medicación sin interrumpirla. pacientes con esta infección, sin poder establecer causalidad (14-17).

El tratamiento con levotiroxina no deberá suspenderse. En los casos de hipertiroidismo, los medicamentos deben continuarse por el riesgo de descompensación aguda al suspenderlos, que puede poner en riesgo la vida y requerir atención intrahospitalaria, incluso en la unidad de cuidado intensivo. Un evento adverso infrecuente del uso de metimazol y el propiltiouracilo es la agranulocitosis (0,5\% de los pacientes) que se caracteriza por neutropenia ( $<1000 / \mathrm{mm} 3$ ); por lo tanto, aquellos pacientes que durante el tratamiento con estos fármacos presenten fiebre u odinofagia deben suspendérseles el medicamento y realizarles un hemograma que permita hacer el diagnóstico diferencial (14-17).

Algunos pacientes con orbitopatía distiroidea reciben esteroides o inmunosupresores. En estos casos las medidas de prevención deben extremarse, dado que están en mayor riesgo de infectarse por COVID-19 y tener enfermedades más severas; dependiendo de la severidad de la enfermedad ocular y de forma individualizada podrá convenirse la suspensión del medicamento o la posibilidad de diferir las dosis (14-17).

Aunque no se ha demostrado que las personas con disfunción tiroidea descompensada tengan mayor riesgo de infecciones virales, es posible que en casos de disfunción tiroidea severa no controlada aumenten las complicaciones; por lo tanto, se recomienda que los pacientes con enfermedad tiroidea continúen tomando su medicación sin interrumpirla; deben seguir las recomendaciones de la población general de higiene de manos y distanciamiento social; cualquier paciente con fiebre, tos o dificultad respiratoria debe buscar atención médica de inmediato (14-17).

\section{Nódulo tiroideo}

Cerca del $70 \%$ de las personas pueden tener uno o más nódulos tiroideos en la ecografía, de ellos alrededor del 90 \% son benignos. Afortunadamente, la mayoría de los tumores tiroideos malignos son de lento crecimiento y buen pronóstico, de modo que diferir la realización de la biopsia del nódulo tiroideo en tiempos de pandemia es seguro, excepto que el médico tratante indique la urgencia del procedimiento $(18,19)$. 
Para el cáncer, la cirugía es el tratamiento de elección y dado que la mayoría de los tumores son de crecimiento muy lento, el riesgo es bajo si la cirugía se retrasa unos meses.

\section{Cáncer de tiroides}

Pocas veces las malignidades en tiroides requieren quimioterapia o tratamientos que afectan el sistema inmune, así que tener un diagnóstico previo de cáncer de tiroides y recibir levotiroxina no es un factor de riesgo para contraer COVID-19 o tener infecciones más severas (15-18,20). A considerar:

- Si se ha recibido terapia con yodo 131 (I131) de forma reciente (menos de 12 semanas) a dosis altas pudiera tenerse mayor riesgo de infección por COVID-19 y enfermedad grave, por la alteración transitoria del sistema inmune; pero si este fue un tratamiento pasado, el riesgo de infección por COVID-19 es igual al de la población general.

- Los pacientes que reciben quimioterapia con inhibidores de la tirosina kinasa se consideran de riesgo de enfermedad grave por COVID-19.

\section{Cirugía de tiroides}

Indicada en enfermedades benignas y malignas. En bocio e hipertiroidismo podrá con seguridad diferirse por algunos meses en tiempos de pandemia, siempre y cuando se mantengan los tratamientos médicos. Para el cáncer, la cirugía es el tratamiento de elección y dado que la mayoría de los tumores son de crecimiento muy lento, el riesgo es bajo si la cirugía se retrasa unos meses. Las cirugías electivas deberán ser pospuestas para minimizar el riesgo de infección por COVID-19. Según la Asociación Americana de Tiroides sólo se deberá considerar cirugía urgente (antes de cuatro semanas) en casos de (21):

- Cánceres de tiroides que son inminentemente potencialmente mortales, presentan una morbilidad significativa relacionada con la invasión local (tráquea, nervio laríngeo recurrente) o exhiben una biología tumoral agresiva (tiempos de duplicación cortos, crecimiento o recurrencia rápida del tumor o enfermedad local-regional rápidamente progresiva).

- Enfermedad de Graves potencialmente mortal o gravemente sintomática que no puede controlarse médicamente.

- Bocio con compromiso sintomático de la vía aérea o con riesgo de pérdida inminente de la vía aérea.

- Biopsia abierta para la confirmación de sospecha clínica de cáncer de tiroides anaplásico o linfoma de tiroides cuando otras medidas de diagnóstico no han sido concluyentes.

- Pacientes embarazadas con trastornos relacionados con la tiroides que son peligrosos para la salud de la madre o el feto y no pueden controlarse médicamente.

\section{Tratamiento con I131}

Esta indicado en hipertiroidismo o cáncer de tiroides, en ambos casos su uso requiere medidas especiales de aislamiento y se debe tener en cuenta que puede alterar transitoriamente el sistema inmune. En el caso de cancer de tiroides se puede aplazar el tratamiento de forma segura hasta máximo 180 días siguientes a la tiroidectomía. En caso de cancer de tiroides con metástasis a distancia queda a discreción del médico tratante decidir el mejor momento de administrarlo según la pandemia. En 
hipertiroidismo puede diferirse mientras se mantiene la enfermedad controlada con el uso de medicamentos y hasta que se considere seguro administrarlo (14-18).

\section{Conclusión}

Al momento, la pandemia por COVID-19 no parece ser más frecuente ni presentarse con mayor severidad en los pacientes con trastornos tiroideos y si bien pueden ocurrir alteraciones de las pruebas tiroideas durante la enfermedad, parecen ser transitorias y no requerir tratamientos específicos. Es fundamental que los enfermos continúen sus tratamientos y podrán diferirse procedimientos como el yodo radiactivo o cirugías hasta que se considere seguro realizarlos.

\section{Fuente de financiación}

Ninguna.

\section{Conflictos de intereses}

Se declara que no existe ningún conflicto de interés en el presente artículo.

\section{Ética de la publicación}

Se trata de una revisión de tema, que no incluye experimentación con animales ni ensayos clínicos.

\section{Bibliografía}

Es fundamental que los enfermos continúen sus tratamientos y podrán diferirse procedimientos como el yodo radiactivo o cirugías hasta que se considere seguro realizarlos.
1. Desailloud R, Hober D. Viruses and thyroiditis: an update. Virol J. 2009;6:5.

2. Yang J, Zheng Y, Gou X, Pu K, Chen Z, Guo Q, et al. Prevalence of comorbidities and its effects in coronavirus disease 2019 patients: A systematic review and meta-analysis. Int J Infect Dis. 12 de marzo de 2020;94:91-5.

3. Wang D, Hu B, Hu C, Zhu F, Liu X, Zhang J, et al. Clinical characteristics of 138 hospitalized patients with 2019 novel Coronavirus-Infected pneumonia in Wuhan, China. JAMA. 7 de febrero de 2020;323(11):1061-1069.

4. Gupta N, Agrawal S, Ish P, Mishra S, Gaind R, Usha G, et al. Clinical and epidemiologic profile of the initial COVID-19 patients at a tertiary care centre in India. Monaldi Arch Chest Dis Arch Monaldi Mal Torace. 10 de abril de 2020;90(1).

5. Henry BM, de Oliveira MHS, Benoit S, Plebani M, Lippi G. Hematologic, biochemical and immune biomarker abnormalities associated with severe illness and mortality in coronavirus disease 2019 (COVID-19): a meta-analysis. Clin Chem Lab Med. 10 de abril de 2020;58 (7):1021-1028.

6. Chen T, Wu D, Chen H, Yan W, Yang D, Chen G, et al. Clinical characteristics of 113 deceased patients with coronavirus disease 2019: retrospective study. BMJ. 26 de 2020;368:m1091.

7. Wang W., Ye Y.X., Yao H. Evaluation and observation of serum thyroid hormone and parathyroid hormone in patients with severe acute respiratory syndrome. $J$ Chin Antituberculous Assoc. 2003;25:232-234. 


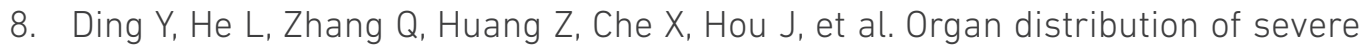
acute respiratory syndrome (SARS) associated coronavirus (SARS-CoV) in SARS patients: implications for pathogenesis and virus transmission pathways. J Pathol. junio de 2004;203(2):622-30.

9. Wei L, Sun S, Xu C, Zhang J, Xu Y, Zhu H, et al. Pathology of the thyroid in severe acute respiratory syndrome. Hum Pathol [Internet]. enero de 2007 [citado 11 de mayo de 2020];38(1):95-102. Disponible en: https://www.ncbi.nlm.nih.gov/pmc/ articles/PMC7112059/

10. Wei L, Sun S, Zhang J, Zhu H, Xu Y, Ma Q, et al. Endocrine cells of the adenohypophysis in severe acute respiratory syndrome (SARS). Biochem Cell Biol [Internet]. 2010 ;88(4):723-30. Disponible en: https://www.nrcresearchpress.com/ doi/10.1139/010-022

11. Ye YX, Wang W, Yao H, Li HM, Sun LQ, Wang AS, et al. Change in hormones of related sexual function in patients with severe acute respiratory syndrome. Labeled Immunoass Clin Med. 2004;11:63-5.

12. Leow MK, Kwek DS, Ng AW, Ong K, Kaw GJ, Lee LS. Hypocortisolism in survivors of severe acute respiratory syndrome (SARS). Clin Endocrinol (Oxf) [Internet]. 2005 [citado 11 de mayo de 2020];63(2):197-202. Disponible en: https://www. ncbi.nlm.nih.gov/pmc/articles/PMC7188349/

13. Chrousos GP, Kaltsas G. Post-SARS sickness syndrome manifestations and endocrinopathy: how, why, and so what? Clin Endocrinol (Oxf) [Internet]. 2005 [citado 11 de mayo de 2020];63(4):363-5. Disponible en: https://www.ncbi.nlm.nih. gov/pmc/articles/PMC7162060/

14. Boelaert K, Visser WE, Taylor PN, Moran C, Léger J, Persani L. ENDOCRINOLOGY IN THE TIME OF COVID-19: Management of hyperthyroidism and hypothyroidism. Eur J Endocrinol. 2020;183(1):G33-9.

15. American Thyroid Association. Novel Coronavirus (COVID-19) and the Thyroid: Frequently Asked Questions [Internet]. American Thyroid Association. [citado 27 de julio de 2020]. Disponible en: https://www.thyroid.org/covid-19/coronavirus-frequently-asked-questions/

16. British Thyroid Foundation. Thyroid disease and coronavirus (COVID-19) [Internet]. British Thyroid Foundation. [citado 27 de julio de 2020]. Disponible en: https://www.btf-thyroid.org/news/thyroid-disease-and-coronavirus-covid-19

17. Sociedad Española de Endocrinología, y Nutrición (SEEN). Posicionamiento del Área de Conocimiento de Tiroides de la Sociedad Española de Endocrinología y Nutrición (SEEN) sobre los efectos de la pandemia de COVID-19 sobre las enfermedades tiroideas [Internet]. Disponible en: https://www.seen.es/ModulGEX/workspace/publico/ modulos/web/docs/apartados/1091/150420 104500 4745077698.pdf

18. Vrachimis A, lakovou I, Giannoula E, Giovanella L. Endocrinology in the time OF COVID-19: Management of thyroid nodules and cancer. Eur J Endocrinol. julio de 2020;183(1):G41-8. 
19. American Thyroid Association. Clinical Affairs Committee: Physician Guidance to Thyroid FNA during COVID-19 crisis [Internet]. American Thyroid Association. [citado 27 de julio de 2020]. Disponible en: https://www.thyroid.org/covid-19/ clinical-committee-physician-guidance/

20. British Thyroid Foundation. Thyroid cancer and coronavirus (COVID-19) [Internet]. British Thyroid Foundation. [citado 27 de julio de 2020]. Disponible en: https://www.btf-thyroid.org/thyroid-cancer-and-coronavirus

21. Metzger R, Singer M. Comment on Thyroid Surgery During the Covid-19 Pandemic [Internet]. American Thyroid Association: Comment on Thyroid Surgery During the Covid-19 Pandemic. [citado 5 de junio de 2020]. Disponible en: https://www. thyroid.org/covid-19/comment-on-thyroid-surgery-during-pandemic/ 